\title{
Potential urine biomarkers for gestational hypertension and preeclampsia
}

\author{
HONG-XIA GUO ${ }^{1}$, YAN-BIN ZHU², CUI-PING WU², MEI ZHONG ${ }^{3}$ and SHUI-WANG HU ${ }^{4}$ \\ ${ }^{1}$ Department of Obstetrics, Baoan Maternal and Child Health Hospital, Jinan University, Shenzhen, \\ Guangdong 518102; ${ }^{2}$ Department of Obstetrics and Gynecology, Shenzhen Hospital, Southern Medical University, Shenzhen, \\ Guangdong 518100; ${ }^{3}$ Department of Obstetrics and Gynecology, Nan Fang Hospital of Southern Medical University; \\ ${ }^{4}$ Department of Pathophysiology, Southern Medical University, Guangzhou, Guangdong 510515, P.R. China
}

Received April 22, 2018; Accepted December 17, 2018

DOI: $10.3892 / \mathrm{mmr} .2019 .9911$

\begin{abstract}
Differential proteomic technology was used to identify urine proteomic profile of gestational hypertension and preeclampsia. Urine samples were collected from 10 patients with gestational hypertension, 10 patients with mild preeclampsia, 10 patients with severe preeclampsia and 10 normal pregnancies and analyzed by 2-D difference gel electrophoresis, then matrix assisted laser desorption ionization mass spectrometry was used to identify differential proteins. Subsequently, ELISA was used to verify the content variation of the identified proteins in 200 urine samples. In total, 30 differential proteins were identified. For prostaglandin-H2 D-isomerase (L-PGDS), perlecan and other 15 proteins, the contents in patients with gestational hypertension were higher than that of normal pregnancies, but lower in mild and severe preeclampsia. By contrast, serum albumin and $\alpha$-1-antitrypsin was lower in samples from patients with gestational hypertension and higher in patients with mild and severe preeclampsia compared with normal pregnancies. ELISA verified that the urinary concentration of L-PGDS and perlecan were significantly lower in patients with preeclampsia than in normal pregnancies $(\mathrm{P}<0.05)$. Urine proteomics is a useful tool to identify potential biomarkers to distinguish between different types of hypertensive disorders in pregnancy. L-PGDS and perlecan could potentially be used as markers to reflect the state of renal function, and may participate in the genesis and development of renal injury during preeclampsia.
\end{abstract}

Correspondence to: Professor Hong-Xia Guo, Department of Obstetrics, Baoan Maternal and Child Health Hospital, Jinan University, 56 Yulv Road, Shenzhen, Guangdong 518102, P.R. China E-mail: doctorghx@163.com

Key words: preeclampsia, body fluid, two-dimensional difference gel electrophoresis, matrix assisted laser desorption ionization-time of flight-mass spectrometry, L-prostaglandin-H2 D-isomerase, perlecan

\section{Introduction}

Hypertensive disorders of pregnancy (HDP) are one of the major causes of maternal mortality around the world. Routine prenatal care decreases the morbidity rate of the pregnancy-specific seizure disorder, eclampsia; however, the rate of preeclampsia, a common and major complication causing significant maternal and fetal morbidity and mortality worldwide (1), remains largely unchanged in China.

A great deal of effort has been directed at the identification of HDP etiology and pathogenesis; however, there is no specific biomarker or test ready for clinical use to make early diagnosis and predict severity, although there are some encouraging findings (2). Based on a century of research into HDP, there is increased understanding of this disease; however, the complete etiology and pathogenesis remain unclear because the causes of HDP are not independent and specific biomarkers and methods are limited. Therefore, identifying a useful measure to make early diagnosis and estimate the severity of HDP is currently an important research hot spot in obstetrics.

Following the development of proteomics, new approaches, including comparative differential proteomics, can provide reliable data with accurate quantitation and good reproducibility. At present there are various novel differential proteomics analysis methods, among which difference gel electrophoresis (DIGE) technology is one of the most popular, as it has high-resolution, inherited from two-dimensional gel electrophoresis (2-DE), and has high reproducibility, high sensitivity, high throughput capability and high dynamic range. DIGE is a method that can label protein samples with different fluorescent dyes prior to 2-DE, and can separate up to three different protein samples at the same time in one two-dimensional gel $(3,4)$. The internal standard is used to match the protein patterns across gels; thus, negating the problem of inter-gel variation, a common issue with 2-DE (4). Recently, this technology has exhibited important value for early diagnosis of malignant tumors, including nasopharyngeal carcinoma, breast cancer, gastric cancer, liver cancer, autoimmune disease and infectious diseases, with specificity and sensitivity up to $90 \%$ (5). This technology has also been used to investigate obstetrical diseases (6). Based on differential proteome analysis of fetal accessory (including placenta, 
membrane, umbilical cord and amniotic fluid) and maternal body fluids, including placenta, amniotic fluid, blood, cerebrospinal fluid and urine, it is possible to investigate the molecular biology of pregnancy and its complications. By comparing the differential proteome of normal pregnancy and those with obstetrical complications, certain potential specific molecular biomarkers may be identified.

Urine is the final metabolic product of blood following glomerular filtration and renal tubule resorption; thus, the change of its composition, quantity and characteristics can reflect the information of urinary system diseases, and even the metabolic condition of the whole body. Though the American College of Obstetricians and Gynecologists no longer consider proteinuria as a diagnostic criteria for preeclampsia (7), the quantity and quality of the protein changes in urine may still be associated with preeclampsia; therefore, the current study aimed to determine the differences in protein content between the urine of patients with HDP and normal pregnancy using a technology platform based on DIGE, matrix assisted laser desorption ionization-time of flight/time of flight mass spectrometry (MALDI TOF/TOF) and bioinformatics analysis. Subsequently, the content changes of identified proteins in the patient urine were validated using ELISA.

\section{Materials and methods}

Materials.Cyanine(Cy)2,Cy3,Cy5,Tris,3-[(3-cholamidopropyl) dimethylammonio]-1-propanesulfonate, tetramethylethylenediamine, dithiothreitol (DTT), EDTA, dimethylformamide, $\mathrm{Ettan}^{\mathrm{TM}}$ 2-D Quant Kit, Ettan ${ }^{\mathrm{TM}}$ 2-D Clean-up Kit were obtained from GE Healthcare (Chicago, IL, USA). Trichloroacetic acid, trifluoroacetic acid and cyano-4-hydroxycinnamic acid were purchased from Sigma-Aldrich (Merck KGaA, Darmstadt, Germany). Human prostaglandin-H2 D-isomerase (L-PGDS; cat. no. RD191113100R) and perlecan (cat. no. DPSG10) ELISA kits were from BioVendor-Laboratorni Medicina A.S. (Brno, Czech Republic).

\section{Sample preparation}

Urine samples were collected from 10 patients with gestational hypertension (Group G), 10 patients with mild preeclampsia (Group M), 10 patients with severe preeclampsia (Group S) and 10 normal pregnancies (Group N) admitted to the Department of Obstetrics, Shenzhen Maternity and Child Healthcare Hospital Affiliated to Southern Medical University (Shenzhen, China) between September 2013 and December 2013, (aged 26-41). Written informed consent was obtained from all study participants and ethical approval for this study was obtained from the local research ethics committee of Southern Medical University. Gestational hypertension is diagnosed by $\mathrm{BP} \geq 140 / 90 \mathrm{mmHg}$ first appearing during pregnancy and returning to normal 12 weeks postpartum without proteinuria. Mild preeclampsia is diagnosed by $\mathrm{BP} \geq 140 / 90 \mathrm{mmHg}$ after 20 gestational weeks and urinary albumin $\geq 0.3 \mathrm{~g} / 24 \mathrm{~h}$ or $(+)$. The diagnosis criteria of severe preeclampsia includes $\mathrm{BP} \geq 160 / 110 \mathrm{mmHg}$ and urinary albumin $\geq 5 \mathrm{~g} / 24 \mathrm{~h}$ or $(++)$, serum creatinine level $\geq 106 \mu \mathrm{mol} / 1$, blood platelet $<100 \times 10^{9} / 1$, elevated lactate dehydrogenase, elevated transaminase, persistent headache or visual disorder, persistent upper abdomen discomfort and fetal growth restriction. Women with a history of hypertension, renal disease, heart disease, liver disease, diabetes, severe anemia, malignant tumor and rheumatic disease were excluded. The clinical data from these 40 cases are presented Table I. A total of 10 urine samples were mixed into one sample separately and then ultrafiltration and centrifugation were performed to obtain the urine protein sample. Ettan $^{\mathrm{TM}}$ 2-D Clean-up kit and Ettan ${ }^{\mathrm{TM}}$ 2-D Quant kit were used to purify and quantify the urine protein sample.

Protein labeling with CyDye DIGE fluor. Urine protein samples were labeled with $\mathrm{Cy} 2, \mathrm{Cy} 3$ and $\mathrm{Cy} 5$ following dissolving in dimethylformamide. Typically, $50 \mu \mathrm{g}$ lysate was labeled with 400 pmol Cy3 and Cy5, while the same amount of the pool standard that contained equal quantities of all the samples was labeled with Cy2, namely the internal label. Each sample was loaded on two gels labeled with Cy3 and Cy5 to achieve statistical confidence. Prior to quenching with $1 \mu 1$ $10 \mathrm{mM}$ lysine for $10 \mathrm{~min}$ on ice, the Cy dye was placed in the dark on ice for $30 \mathrm{~min}$ to acquire labeling reactions. These labeled samples were then combined for 2-D DIGE analysis.

2-DE. 2-DE was performed with Ettan ${ }^{\mathrm{TM}}$ IPGPhor and Ettan $^{\text {TM }}$ DALT Six electrophoresis units. Prior to SDS-PAGE, each strip was equilibrated with $10 \mathrm{ml}$ equilibration buffer A (6 M urea, $50 \mathrm{mM}$ Tris-HCL pH 8.8, 30\% glycerol, 2\% SDS, $10 \mathrm{mg} / \mathrm{ml} \mathrm{DTT}$ ) on a rocking table for $15 \mathrm{~min}$, followed by $10 \mathrm{ml}$ equilibration of buffer B (6 M urea, $50 \mathrm{mM}$ Tris-HCL $\mathrm{pH} 8.8,30 \%$ glycerol, $2 \%$ SDS, $25 \mathrm{mg} / \mathrm{ml}$ iodoacetamide) for another $15 \mathrm{~min}$. Then the trips were loaded and run on $12 \%$ acrylamide isocratic Laemmli gels with running parameter settings as constant power of $3 \mathrm{~W}$ per gel for $45 \mathrm{~min}$, followed by $17 \mathrm{~W}$ per gel until the bromophenol blue dye front had run off the bottom of the gels. Then, the Typhoon 9410 imager (GE Healthcare) was used to visualize the labeled protein and match with DIGE images with DeCyder 6.5 software (GE Healthcare) to identify the spots of interest.

MALDI TOF/TOF and protein identification. Protein spots of interest were excised using an automatic Spot picker, and then the picked spots underwent in-gel digestion, followed by analysis with ABI 4800 MALDI TOF/TOF mass spectrometry (Applied Biosystems; Thermo Fisher Scientific, Inc., Waltham, MA, USA). The mass spectral data were submitted to the SWISSPROT database (www.uniprot.org/) using Mascot software (www.matrixscience.com). The proteins with identification probabilities of $>95 \%$ given by Mascot were considered successfully identified and then received bioinformatics analysis.

ELISA analysis. From the identified proteins, two were selected, L-PGDS and perlecan, to be analyzed by ELISA to confirm the content change in urine. Urine samples were collected from another 50 patients with gestational hypertension (Group G), 50 patients with mild preeclampsia (Group M), 50 patients with severe preeclampsia (Group S) and 50 normal pregnancies (Group N) admitted to Department of Obstetrics, Shenzhen Maternity and Child Healthcare Hospital Affiliated to Southern Medical University (Shenzhen, China), between January 2014 and December 2014. Similarly, written informed 
Table I. Patient clinical information.

\begin{tabular}{|c|c|c|c|c|c|c|}
\hline Characteristic & Group N & Group G & Group M & Group S & F-value & P-value \\
\hline Age (years) & $32.58 \pm 4.43$ & $33.41 \pm 4.56$ & $31.72 \pm 5.12$ & $34.02 \pm 3.96$ & 0.243 & 0.865 \\
\hline Gravity & $2.38 \pm 1.27$ & $2.58 \pm 1.13$ & $2.43 \pm 1.21$ & $2.41 \pm 1.34$ & 0.026 & 0.994 \\
\hline Parity & $0.68 \pm 0.26$ & $0.75 \pm 0.17$ & $0.73 \pm 0.21$ & $0.67 \pm 0.25$ & 0.147 & 0.93 \\
\hline Gestational age (days) & $277.75 \pm 18.27$ & $274.36 \pm 20.15$ & $269.56 \pm 22.35$ & $265.84 \pm 20.75$ & 0.329 & 0.804 \\
\hline
\end{tabular}

Group N, normal pregnancies; Group G, gestational hypertension; Group M, mild preeclampsia; Group S, severe preeclampsia.

consent was obtained from all study participants and ethical approval for this study was obtained from the local research ethics committee of Shenzhen Hospital, Southern Medical University (Shenzhen, China). The inclusion criteria were consistent with the diagnosis criteria. Women with a history of hypertension, renal disease, heart disease, liver disease, diabetes, severe anemia, malignant tumor and rheumatic disease were excluded. According to the kit (BioVendor-Laboratorni Medicina A.S.), the urine concentration of L-PGDS and perlecan were determined in all patients.

Statistical analysis. Data were presented as the mean \pm standard deviation. The one-way analysis of variance and further multiple comparisons by Student-Newman-Keuls test were used for comparing the L-PGDS and perlecan between Group N, G, N and M. SPSS (version 19.0; IBM Corp., Armonk, NY, USA) was used for statistical analysis. $\mathrm{P}<0.05$ was considered to indicate the statistically significant difference.

\section{Results}

Results of 2-DE and mass spectrometry. Total proteins were extracted from the urine of 10 patients with gestational hypertension, 10 patients with mild preeclampsia, 10 patients with severe preeclampsia and 10 normal pregnancies, and then $50 \mu \mathrm{g}$ samples of the four groups subjected to SDS-PAGE electrophoresis for accuracy (Fig. 1). The 2-DE images were obtained by fluorescence scanning and analyzed using DeCyder software to estimate the abundance of proteins in each sample objectively to provide quantitative data. A filter threshold of 1.5 was set to detect the protein spots of interest that had a significant change among the four groups. Compared with the normal pregnancy group, 44 differential protein spots were identified, among which 22 were upregulated and 22 were downregulated in the gestational hypertension group, 15 were upregulated and 30 downregulated in the mild preeclampsia group compared with the gestational hypertension group. There were 45 differential protein spots when comparing the severe preeclampsia group with the mild preeclampsia group, of which 9 were upregulated and 36 were downregulated. On account of some protein spots changing in all four groups, 65 differential protein spots were analyzed in total (Fig. 2).

Following mass spectrometry analysis, 30 differential proteins were identified. Ceruloplasmin, Ig- $\gamma-3$ chain $\mathrm{C}$ region and pancreatic $\alpha$-amylase were all upregulated in hypertensive disorders in pregnancy; however, hemopexin, antithrombin-III, kininogen-1, $\alpha$-2-HS-glycoprotein, vitamin D-binding protein, actin cytoplasmic 2, apolipoprotein A-I and

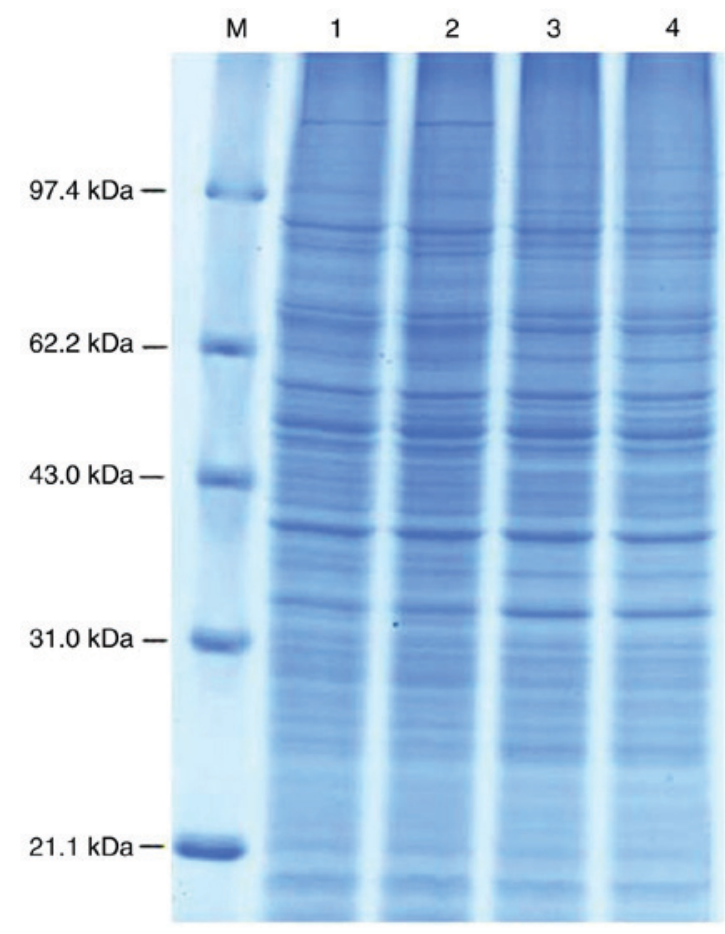

Figure 1. SDS-PAGE image of total protein from urinary samples. M, protein marker; 1 , total urinary protein from normal pregnancy group; 2 , total urinary protein from gestational hypertension group; 3 , total urinary protein from mild preeclampsia group; 4 , total urinary protein from severe preeclampsia group.

chorionic somatomam-motropin hormone were all downregulated. For $\alpha$-1B-glycoprotein, cadherin-11, pregnancy-specific $\beta$-1-glycoprotein 11 , gelsolin, inter- $\alpha$-trypsin inhibitor heavy chain $\mathrm{H} 4$, keratin type II cytoskeletal 2 epidermal, protein $\alpha-1$-microglobulin/bikunin precursor (AMBP), vesicular integral-membrane protein VIP36, filamin-A-interacting protein 1, semenogelin-1, L-PGDS, Ig $\kappa$ chain V-III region WOL, perlecan, mannan-binding lectin serine protease 2, fibrinogen alpha chain and long palate, lung and nasal epithelium carcinoma-associated protein 1 contents were higher in gestational hypertension than in normal pregnancies, but lower in mild and severe preeclampsia. By contrast, serum albumin and $\alpha$-1-antitrypsin were lower in gestational hypertension compared with normal pregnancies, and higher in mild and severe preeclampsia. No significant difference was observed in the remaining protein, ganglioside GM2 activator, in the four groups. The genes and functions of all these identified proteins are presented in Table II.

Using the DeCyder software, abundance curves of these differential protein spots, no. 1,618 and no. 1,911, which can 


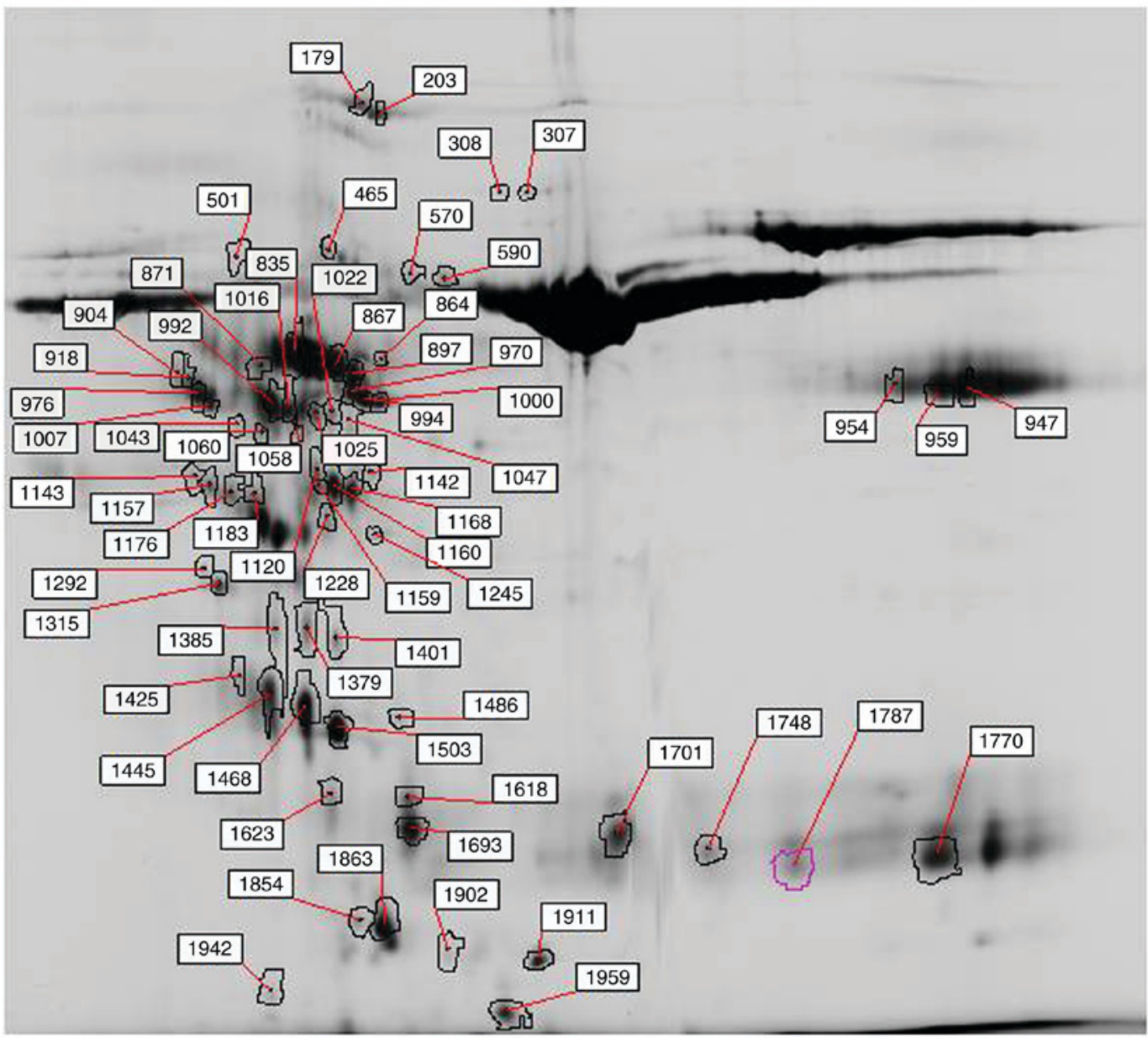

Figure 2. Distribution of the 65 differentially expressed protein spots after analysis by DeCyder 2D software.

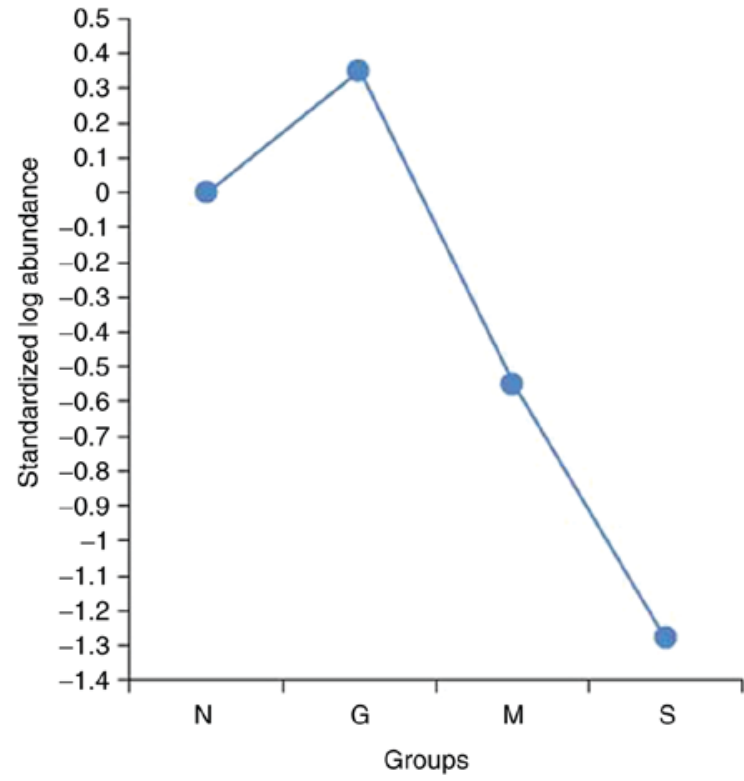

Figure 3. Abundance curve of no. 1,618 in the four groups. N, normal pregnancies; $\mathrm{G}$, gestational hypertension; $\mathrm{M}$, mild preeclampsia; $\mathrm{S}$, severe preeclampsia.

reflect the state of renal function were simulated (Figs. 3 and 4). The abundance ratio of gestational hypertension and normal pregnancies for spot no. 1,618 was 2.13 , and the ratio was 1.58

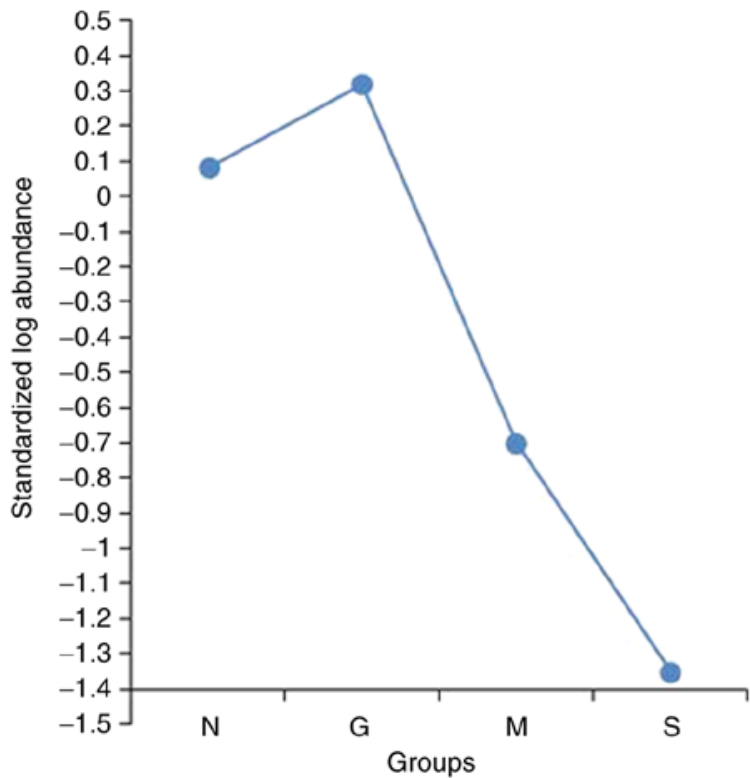

Figure 4. Abundance curve of no. 1,911 in the four groups. N, normal pregnancies; G, gestational hypertension; M, mild preeclampsia; S, severe preeclampsia.

for spot no. 1911. However, for mild and severe preeclampsia, the abundance curve exhibited reductions in spot no. 1,618 and no. 1,911 , with ratios of 0.26 and 0.16 in mild preeclampsia, 


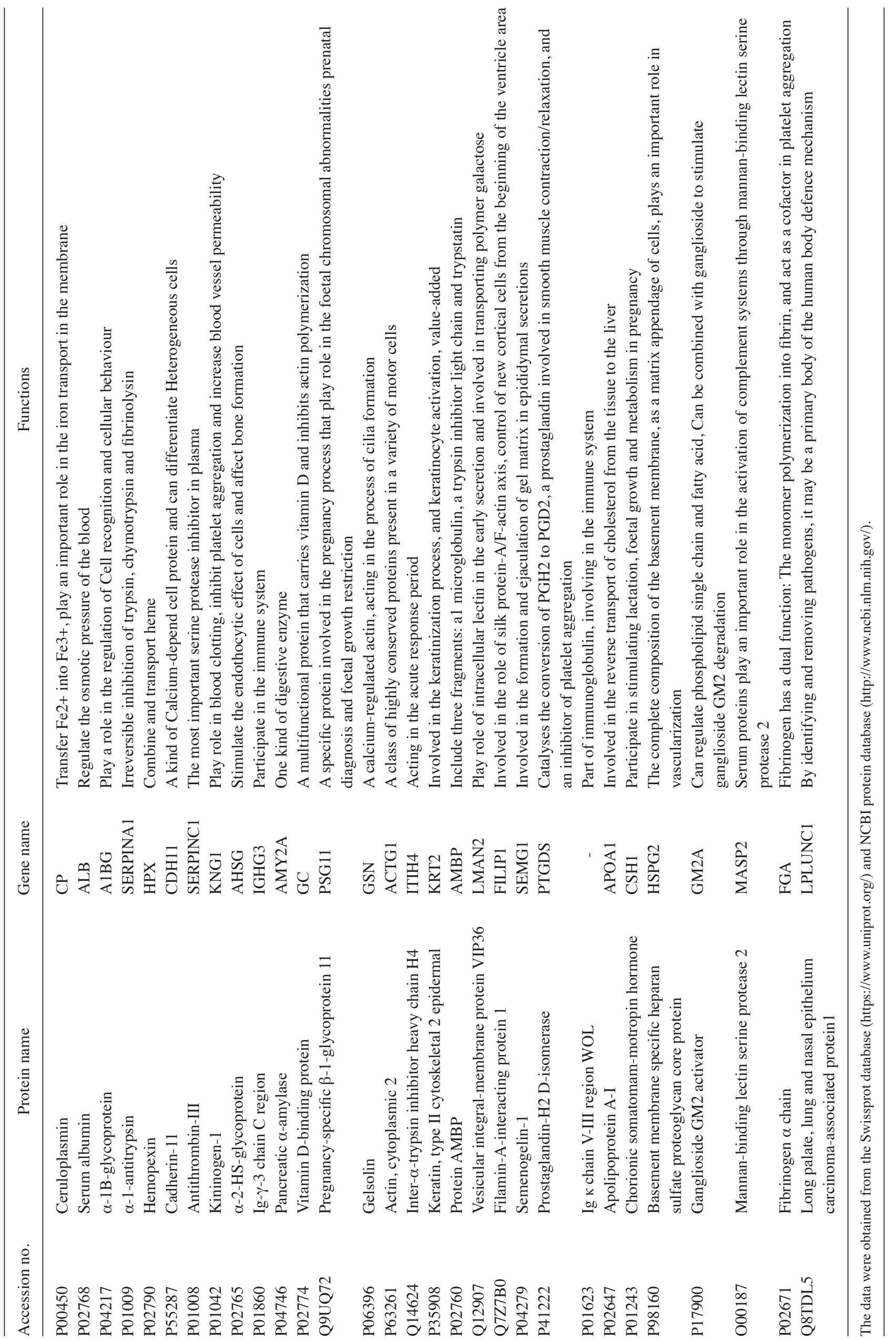


Table III. ELISA results of L-PGDS and perlecan in the four patient groups.

\begin{tabular}{lcccrr}
\hline Protein & Group N & Group G & Group M & Group S & F-value \\
\hline L-PGDS $(\mu \mathrm{g} / \mathrm{ml})$ & $1.26 \pm 0.25^{\mathrm{a}-\mathrm{c}}$ & $1.32 \pm 0.16^{\mathrm{b}, \mathrm{c}}$ & $0.94 \pm 0.23^{\mathrm{c}}$ & $0.78 \pm 0.23$ & 68.59 \\
Perlecan $(\mathrm{nmol} / \mathrm{l})$ & $9.99 \pm 1.85^{\mathrm{b}-\mathrm{d}}$ & $12.73 \pm 3.71^{\mathrm{b}, \mathrm{c}}$ & $8.02 \pm 1.66^{\mathrm{c}}$ & $6.63 \pm 1.39$ & 64.01 \\
\hline
\end{tabular}

${ }^{\mathrm{a}} \mathrm{P}>0.05$ vs. Group $\mathrm{G}$; ${ }^{\mathrm{b}} \mathrm{P}<0.05$ vs. Group $\mathrm{M} ;{ }^{\mathrm{c}} \mathrm{P}<0.05$ vs. Group $\mathrm{S} ;{ }^{\mathrm{d}} \mathrm{P}<0.05$ vs. Group G. Group N, normal pregnancies; Group G, gestational hypertension; Group M, mild preeclampsia; Group S, severe preeclampsia; L-PGDS, prostaglandin-H2 D-isomerase.

respectively, and 0.06 and 0.04 in severe preeclampsia, respectively. According to mass spectrometry analysis and database searching, spot no. 1,618 was confirmed as L-PGDS and no. 1911 was perlecan. In consideration of their change trends, the two proteins may have important effects in the genesis of gestational hypertension and preeclampsia.

Results of ELISA. To validate the results regarding L-PGDS and perlecan, ELISA was used for further confirmation in another 50 patients with gestational hypertension (Group G), 50 patients with mild preeclampsia (Group M), 50 patients with severe preeclampsia (Group $\mathrm{S}$ ) and 50 normal pregnancies (Group N). When observing the changes in L-PGDS, there was significant difference among the four groups and further multiple comparisons indicated that there was no variation between Group N $(1.26 \pm 0.25 \mu \mathrm{g} / \mathrm{ml})$ and Group G $(1.32 \pm 0.16 \mu \mathrm{g} / \mathrm{ml})$; however, the concentration of L-PGDS in Group M $(0.94 \pm 0.23 \mu \mathrm{g} / \mathrm{ml})$ and Group S $(0.78 \pm 0.23 \mu \mathrm{g} / \mathrm{ml})$ were significantly lower than that of Group $\mathrm{N}$ and Group $\mathrm{G}$ (both $\mathrm{P}<0.001$ ). When comparing Group $\mathrm{M}$ with Group $\mathrm{S}$, there were significantly lower levels in Group $\mathrm{S}(\mathrm{P}=0.023)$. Regarding the urine perlecan concentration, the results illustrated a higher concentration in Group G $(12.73 \pm 3.71 \mathrm{nmol} / \mathrm{l}$; $\mathrm{P}=0.036)$ and lower in Group $\mathrm{M}(8.02 \pm 1.66 \mathrm{nmol} / \mathrm{l}, \mathrm{P}=0.006)$ and Group S $(6.63 \pm 1.39 \mathrm{nmol} / 1, \mathrm{P}<0.001)$ compared with Group N $(9.99 \pm 1.85 \mathrm{nmol} / \mathrm{l})$. Similarly, perlecan was decreased in Group S compared with Group M ( $\mathrm{P}=0.039$; Table III).

\section{Discussion}

With the development of DIGE and MALDI TOF/TOF technologies, proteomic research has been widely used in the post-genomics era, and can provide new opportunities to identify the novel biomarkers for various important diseases, as it allows for the large-scale investigation of proteins in complex biological fluids and tissues in a high-throughput manner. The proteomic studies of preeclampsia have predominantly focused on maternal blood, placenta, amniotic fluid and trophoblast cells $(3,4)$. When comparing the levels the low molecular-weight protein in serum from patients with preeclampsia in the early second trimester with a control group, Anand et al (8) identified $>60$ potential biomarkers, which when modeled in a 14 different multi-marker combinations exhibited sensitivity and specificity of $>90 \%$ for the early detection of women at risk for preeclampsia. Another proteomic study on preeclampsia indicated that $\mathrm{C} 1 \mathrm{~s}$ subcomponent and protein AMBP were significantly overexpressed in the preeclamptic serum (9), which may be useful in early prediction.
Although urine is a good source of biomarkers, there few studies have researched preeclampsia using urine samples and no consistent results have been found (10). Carty et al (11) used a proteomic strategy to identify urinary biomarkers that predict preeclampsia prior to the onset of disease and screened out several biomarkers, including fibrinogen $\alpha$ chain, collagen $\alpha$ chain and uromodulin fragments, which may contribute to better prediction, monitoring and accurate diagnosis of preeclampsia.

Proteinuria is used to distinguish between gestational hypertension and preeclampsia; therefore, the quantity and component changes of urine protein may have important role in distinction of these two types of hypertensive disorders of pregnancy $(12,13)$. Differential proteins were examined in the urine of patients with gestational hypertension, mild preeclampsia, severe preeclampsia and normal pregnancies using DIGE and mass spectrometry, and 30 differential proteins were identified, among which $\alpha-1 \mathrm{~B}$-glycoprotein, cadherin-11, pregnancy-specific $\beta-1$-glycoprotein 11 , gelsolin, inter- $\alpha$-trypsin inhibitor heavy chain $\mathrm{H} 4$, keratin type II cytoskeletal 2 epidermal, protein AMBP, vesicular integral-membrane protein VIP36, filamin-A-interacting protein 1, semenogelin-1, mannan-binding lectin serine protease 2, fibrinogen $\alpha$ chain and long palate, lung, nasal epithelium carcinoma-associated protein 1 , Ig $\kappa$ chain V-III region WOL, L-PGDS and perlecan were overexpressed in gestational hypertension, and expression was reduced in patients with mild and severe preeclampsia compared with that of normal pregnancies. All these proteins have their own functions and may have certain effects on the disease; information on their functions is presented in Table III.

L-PGDS ( $\beta$ trace protein) has anionic charge, is of smaller molecular weight than serum albumin (26,000 vs. $66,000 \mathrm{Da})$ and catalyzes prostaglandin $\mathrm{H} 2$ conversion into prostaglandin D2. L-PGDS is distributed in the central nervous system, visual system, male genital system and cardiovascular system. L-PGDS is synthesized in the choroid plexus or leptomeninges of the brain and secreted steadily through cerebrospinal fluid into circulating blood. Therefore it can be detected in cerebrospinal fluid, aqueous fluid, blood plasma, urine and amnio fluid. As serum L-PGDS is excreted through glomerular capillary walls, reduction in the number of functioning glomeruli decreases the renal clearance of L-PGDS and increases serum L-PGDS concentrations. Urinary L-PGDS excretions may be more useful to predict the increased glomerular permeability in an early stage of systemic diseases because of the larger molecular weight and its anionic property $(14,15)$. In the current study, the concentration of urinary L-PGDS was decreased in preeclampsia, particularly in severe 
preeclampsia, indicating that the renal function was altered in these patients. However, an opposing view can be found in which urinary L-PGDS excretions are increased in patients with various forms of renal diseases (16). Therefore, in the future transgenic L-PGDS knock-out mouse models should be used to verify its function in preeclampsia. Additionally, the product produced by L-PGDS, prostaglandin D2, which inhibits platelet aggregation and causes relaxation of vascular smooth muscle, may have a role in the genesis of preeclampsia development (17).

Perlecan is a basement membrane-specific heparan sulfate proteoglycan core protein and is one of the largest proteoglycans, with a protein core of $\sim 500 \mathrm{kDa}$ that can be modified by the addition of N-terminal heparan sulfate side chains. Perlecan is particularly abundant in basement membrane and pericelluar spaces, where it regulates diverse cellular processes, including bone formation, inflammation, cardiac development and angiogenesis. Located at the glomerular basement membrane and glomerular endothelial surface layer, perlecan is the major component of the filtration barrier and has an important role in maintaining renal function. The N-terminal heparan sulfate side chains of perlecan act as a reservoir for growth factors, which can promote angiogenesis by presenting growth factors to their respective cell surface receptors $(18,19)$. By contrast, perlecan can inhibit the autophagy of cells (18). The result of the current study demonstrated that perlecan was decreased in the urine of patients with preeclampsia compared with normal pregnancies, and may indicate the existence of glomerular filtration barrier injury resulting in proteinuria and renal dysfunction, which supports previous research (20). The downregulated expression perlecan in renal organ of different renal diseases and models explains the pathogenesis of renal injury caused not only by glomerular filtration barrier injury but also by the decrease of angiogenesis and the increase of autophagy accompanied the decrease of perlecan. All these evidence supported that perlecan may have effect in the renal change of preeclampsia. Future work will likely ensure this effect using gene knock-out model.

In conclusion, compared with normal pregnancies, differential proteins were identified in the urine of patients with gestational hypertension and preeclampsia. The identified proteins may be good biomarkers for predicting and diagnosing the hypertensive disorders in early pregnancy. L-PGDS and perlecan may be involved in the genesis and development of renal injury in preeclampsia. Further studies will be performed to investigate the function of L-PGDS and perlecan in preeclampsia.

\section{Acknowledgements}

No applicable.

\section{Funding}

This study was funded by research project of Shenzhen Health and Family Planning Commission (grant no. 201605011), Seedling Program of Shenzhen Hospital of Southern Medical University (grant no. 2016MM09) and Natural Science Foundation of China (grant no. 81701473).

\section{Availability of data and materials}

The datasets used or analysed during the current study are available from the corresponding author on reasonable request.

\section{Authors' contributions}

HXG designed the study, performed the research, analysed data and wrote the paper. YBZ performed research and analyzed data. CPW performed research and wrote the paper. MZ helped designed the study and checked the data. SWH performed research and analyzed data.

\section{Ethics approval and consent to participate}

Written informed consent was obtained from all study participants and ethical approval for this study was obtained from the local research ethics committee of Southern Medical University.

\section{Patient consent for publication}

Not applicable.

\section{Competing interests}

The authors declare that they have no competing interests.

\section{References}

1. Roberts JM: Pregnancy related hypertension. In: Maternal Fetal Medicine. Creasy RK and Resnik R (eds). 4th Edition. W.B. Saunders, Philadelphia, PA, pp833-872, 1998.

2. Frampton GK, Jones J, Rose M and Payne L: Placental growth factor (alone or in combination with soluble fms-like tyrosine kinase 1) as an aid to the assessment of women with suspected pre-eclampsia: Systematic review and economic analysis. Health Technol Assess 20: 1-160, 2016.

3. Pasquali M, Serchi T, Planchon S and Renaut J: 2D-DIGE in proteomics. Methods Mol Biol 1654: 245-254, 2017.

4. Alban A, David SO, Bjorkesten L, Andersson C, Sloge E, Lewis S and Currie I: A novel experimental design for comparative two-dimensional gel analysis: Two-dimensional difference gel electrophoresis incorporating a pooled internal standard. Proteomics 3 : 36-44, 2003.

5. Wei YS, Zheng YH, Liang WB, Zhang JZ, Yang ZH, Lv ML, Jia $J$ and Zhang L: Identification of serum biomarkers for nasopharyngeal carcinoma by proteomic analysis. Cancer 112: 544-551, 2008.

6. Vuadens F, Benay C, Crettaz D, Gallot D, Sapin V, Schneider P, Bienvenut WV, Lémery D, Quadroni M, Dastugue B and Tissot JD: Identification of biologic markers of the premature rupture of fetal membranes: Proteomic approach. Proteomics 3: $1521-1525,2003$

7. Roberts JM, August PA, Bakris G, Barton JR, Bernstein IM, Druzin M, Gaiser RR, Granger JP, Jeyabalan A, Johnson DD, et al; American College of Obstetricians and Gynecologists; Task Force on Hypertension in Pregnancy. Hypertension in pregnancy. Report of the American College of Obstetricians and Gynecologists' Task Force on Hypertension in Pregnancy. Obstet Gynecol 122: 1122-1131, 2013.

8. Anand S, Bench Alvarez TM, Johnson WE, Esplin MS, Merrell K, Porter TF and Graves SW: Serum biomarkers predictive of pre-eclampsia. Biomark Med 9: 563-575, 2015.

9. Kim SM, Cho BK, Kang MJ, Norwitz ER, Lee SM, Lee J, Park CW, Kim BJ, Jun JK, Park JS and Yi EC: Expression changes of proteins associated with the development of preeclampsia in maternal plasma: A case-control study. Proteomics 16: 1581-1589, 2016. 
10. Kolialexi A, Mavreli D, Tounta G, Mavrou A and Papantoniou N: Urine proteomic studies in preeclampsia. Proteomics Clin Appl 9: 501-506, 2015.

11. Carty DM, Siwy J, Brennand JE, Zürbig P, Mullen W, Franke J, McCulloch JW, Roberts CT, North RA, Chappell LC, et al: Urinary proteomics for prediction of preeclampsia. Hypertension 57: 561-569, 2011.

12. Buhimschi IA, Zhao G, Funai E, Saade GR and Buhimschi C: Proteomics analysis of urine in preeclampsia (PE): A novel diagnosis for an old disease. Am J Obstet Gynecol 193: S15, 2005.

13. Blankley RT, Gaskell SJ, Whetton AD, Dive C, Baker PN and Myers JE: A proof-of-principle gel-free proteomics strategy for the identification of predictive biomarkers for the onset of pre-eclampsia. BJOG 116: 1473-1480, 2009.

14. Uehara Y, Makino H, Seiki K and Urade Y; L-PGDS Clinical Research Group of Kidney: Urinary excretions of lipocalin-type prostaglandin D synthase predict renal injury in type-2 diabetes: A cross-sectional and prospective multicentre study. Nephrol Dial Transplant 24: 475-482, 2009.

15. Gerhardt T, Pöge U, Stoffel-Wagner B, Klein B, Klehr HU, Sauerbruch T and Woitas RP: Serum levels of beta-trace protein and its association to diuresis in haemodialysis patients. Nephrol Dial Transplant 23: 309-314, 2008.
16. Bacci MR, Cavallari MR, de Rozier-Alves RM, Alves Bda C and Fonseca FL: The impact of lipocalin-type-prostaglandin-D-synthase as a predictor of kidney disease in patients with type 2 diabetes. Drug Des Devel Ther 22: 3179-3182, 2015.

17. Saito S, Tsuda H and Michimata T: Prostaglandin D2 and reproduction. Am J Reprod Immunol 47: 295-302, 2002.

18. Gubbiotti MA, Neill T and Iozzo RV: A current view of perlecan in physiology and pathology: A mosaic of functions. Matrix Biol 57-58: 285-298, 2017.

19. Smith S and Hassell JR: Focus on molecules: Perlecan (HSPG2). Exp Eye Res 83: 471-472, 2006.

20. Conde-Knape K: Heparan sulfate proteoglycans in experimental models of diabetes: A role for perlecan in diabetes complications. Diabetes Metab Res Rev 17: 412-421, 2001.

This work is licensed under a Creative Commons Attribution-NonCommercial-NoDerivatives 4.0 International (CC BY-NC-ND 4.0) License. 